\title{
Changes to central visual fields in cases of severe myopia in a Chinese population
}

\author{
Qian Cao ${ }^{1 \#}$, Lan $\mathrm{Li}^{1 \#}$, Hua Zhong ${ }^{2}$, Tao Wei ${ }^{3}$, Yuan-Sheng Yuan ${ }^{2}$, Yong $\mathrm{Li}^{1}$ \\ ${ }^{1}$ Department of Ophthalmology, The Calmette Affiliated Hospital of Kunming Medical University, Kunming, China; ${ }^{2}$ Department of \\ Ophthalmology, The First Affiliated Hospital of Kunming Medical University, Kunming, China; ${ }^{3}$ Information Consultation Section of Library, \\ Kunming Medical University, Kunming, China \\ Contributions: (I) Conception and design: Q Cao, YS Yuan; (II) Administrative support: L Li, Y Li; (III) Provision of study materials or patients: L \\ Li, Y Li; (IV) Collection and assembly of data: Q Cao; (V) Data analysis and interpretation: Q Cao, H Zhong, T Wei; (VI) Manuscript writing: All \\ authors; (VII) Final approval of manuscript: All authors. \\ \#These authors contributed equally to this work. \\ Correspondence to: Hua Zhong, MD, PhD. Department of Ophthalmology, The First Affiliated Hospital of Kunming Medical University, 295 Xichang \\ Road, Wuhua District, Kunming 650000, China. Email: zhognhua30@163.com.
}

\begin{abstract}
Background: Severe myopia (SM) is commonly defined as a refractive error that exceeds -10.0 diopters (D). To date, however, studies focusing on visual field changes in eyes affected by SM remain scarce. The present study aims to characterize changes to central visual fields in the presence of SM among Chinese individuals and to provide evidence for enabling early diagnosis of primary open-angle glaucoma (POAG) in cases of SM. Methods: The data of 26 cases (44 eyes) involving Chinese individuals with SM were collected using a Humphrey Visual Field Analyzer 750i. The following aspects were identified: types of visual field defects in the probability map of pattern standard deviation, the features of patterns in visual field defects in four quadrants, the frequencies of scotomas, and the proportions of relative scotomas.

Results: (I) Among all visual field defects, 95.45\% were found to be relevant to selected ocular fundus changes; (II) the proportion of relative scotomas was $65.02 \%$. The two major types of visual field scotomas were the enlargement of the physiologic blind spot (43.20\%) and central scotoma (38.64\%), while additional types included nasal step defects $(18.18 \%)$, paracentral scotomas (13.64\%), and temporal sector-shaped defects $(9.09 \%)$. The proportions for scotomas in the four quadrants were: $38.74 \%$ (superior temporal quadrant), $25.87 \%$ (inferior temporal quadrant), 19.32\% (superior nasal quadrant), and 16.07\% (inferior nasal quadrant).

Conclusions: (I) Visual field analysis should be conducted alongside consideration of fundus changes. The bulk of visual field defects were found to be relative scotomas. (II) Scotomas in SM patients were mainly due to the enlargement of a physiological blind spot and central scotomas, and nasal arch-shaped scotomas presented as arc-shaped damage. (III) Abnormal scotomas were detected most frequently in the inferior temporal quadrant.
\end{abstract}

Keywords: Severe myopia (SM); visual field; central; Chinese population

Submitted Dec 12, 2019. Accepted for publication Aug 05, 2020.

doi: 10.21037/apm-19-605

View this article at: http://dx.doi.org/10.21037/apm-19-605

\section{Introduction}

High myopia (HM), defined by a refractive error of at least -5.00 diopters (D) or $-6.00 \mathrm{D}$, has increasingly been highlighted as a public health problem in many countries. It was estimated that by 2050, almost one billion people will be affected by HM (1). Severe myopia (SM), which is commonly defined as a refractive error exceeding $-10.0 \mathrm{D}$, is a more severe form of myopia (2). Primary open-angle glaucoma 
(POAG) is a heterogeneous condition characterized by progressive degeneration of the optic nerve, which may lead to irreversible blindness, and is often associated with elevated intraocular pressure (IOP) (1). Myopia and POAG are both complex eye diseases. Multiple epidemiologic studies have shown that patients with HM, and particularly those with SM, have an increased risk of developing POAG (3). Furthermore, several population-based studies have also shown that the risk of developing POAG increases with the severity of myopia. However, it is important to note that HM eyes show anatomic and structural changes that are clinically extremely challenging to distinguish from those observed in POAG, which may lead to overdiagnosis of POAG in myopic patients. It was reported that this misdiagnosed rate could be as high as $50 \%$ for cases of HM with POAG $(4,5)$. To date, studies on visual field changes in SM eyes remain rare. Accordingly, we carried out the present study to investigate the properties of visual field changes in SM eyes and to provide evidence for the early diagnosis of POAG in cases of SM. We present the following article in accordance with the STROBE Reporting Checklist (available at http://dx.doi. org/10.21037/apm-19-605).

\section{Methods}

A total of 26 SM patients (44 eyes) who visited the Department of Ophthalmology at the First Affiliated Hospital of Kunming Medical University between January 2008 and January 2011 were enrolled in this study. Based on the degree of fundus changes, these patients were categorized into four groups: group I, normal fundus or tigroid fundus (eight eyes); group II, similar to group I + posterior scleral staphyloma (14 eyes); group III, similar to group II + lacquer crack lesion; group IV, similar to group III + Fuchs spot + extensive posterior atrophy of the retina and choroid (15 eyes). The study was approved by the Institutional Review Board of the Ethics Committee of Kunming Medical University and conducted in accordance with the Declaration of Helsinki (as revised in 2013). A written informed consent was obtained from all participants (or their legal guardians).

Patients with primary glaucoma, secondary glaucoma, other eye diseases that may influence IOP and the optic nerve, severe opacity of refractive media, obvious systemic disease, or intracranial disorders were excluded from the present study.

Objective and subjective refractive examinations were performed by the same optometrist. Ophthalmic ultrasound
A and B scanners were used for the eyeball biological measurements. A Goldmann applanation tonometer was employed to test IOP. These tests were all conducted three times. The same ophthalmologist examined the ocular fundus with a slit lamp and performed the fundus photography.

The Humphrey Visual Field Analyzer 750i was employed using a diagnostic accuracy matrix of 24-2, and the SITA standard testing strategy was used for perimetry. All participants had experienced computerized perimetry more than twice. Correcting lenses were used according to the age and diopters of participants, and the results were identified and printed based on single visual field analysis.

\section{Results}

\section{Participant characteristics}

The current study included 26 SM patients (44 eyes). Among them, 11 patients were male (18 eyes) and 15 patients were female (26 eyes). Among the 44 eyes, 21 were right eyes and 23 were left eyes. Additional information about the participants included: their age, which ranged from $14-78$ years old $(42 \pm 14.19$ years old); diopters were between -28.00 and $-10.00 \mathrm{D}(-13.78 \pm 4.69 \mathrm{D})$; the optimal correction vision ranged from $20 / 200$ to $20 / 20$, or between 0.1 and $1.0(0.57 \pm 0.33)$; the optical axial lengths ranged from 24.61 to $32.85 \mathrm{~mm}(27.76 \pm 1.97 \mathrm{~mm})$.

\section{Comparison between ocular fundus lesions and changes in visual fields}

As shown in Table 1, relevant ocular fundus changes were consistent with the visual field defects in 42 of 44 eyes $(95.45 \%)$. The details regarding the typical figures are presented in Figures 1-3. In two eyes, the ocular fundus changes did not completely match the visual field defects.

\section{Morphology of scotomas in SM in different groups of ocular fundus changes}

Among the types of scotomas present in the visual field, physiological blind spots and central scotomas were the two major types, followed by nasal step defects, paracentral scotomas, and temporal sector-shaped defects. The archshaped defect was found in only one case. The details of these findings are presented in Table 2.

Among the 44 eyes, 13 had multiple visual field defects, 
Table 1 Comparison between ocular fundus lesions and changes of visual fields

\begin{tabular}{lccccc}
\hline \multirow{2}{*}{ Results } & \multicolumn{5}{c}{ Groups of ocular fundus changes } \\
\cline { 2 - 6 } & I & II & III & IV & 15 \\
\hline Cases & 8 & 14 & 7 & 15 & 44 \\
Consistent & 6 & 14 & 7 & 0 & $42(95.45 \%)$ \\
Not consistent & 2 & 0 & 0 & $2(4.55 \%)$ \\
\hline
\end{tabular}

Fisher's Exact test: $\mathrm{P}=0.052$.

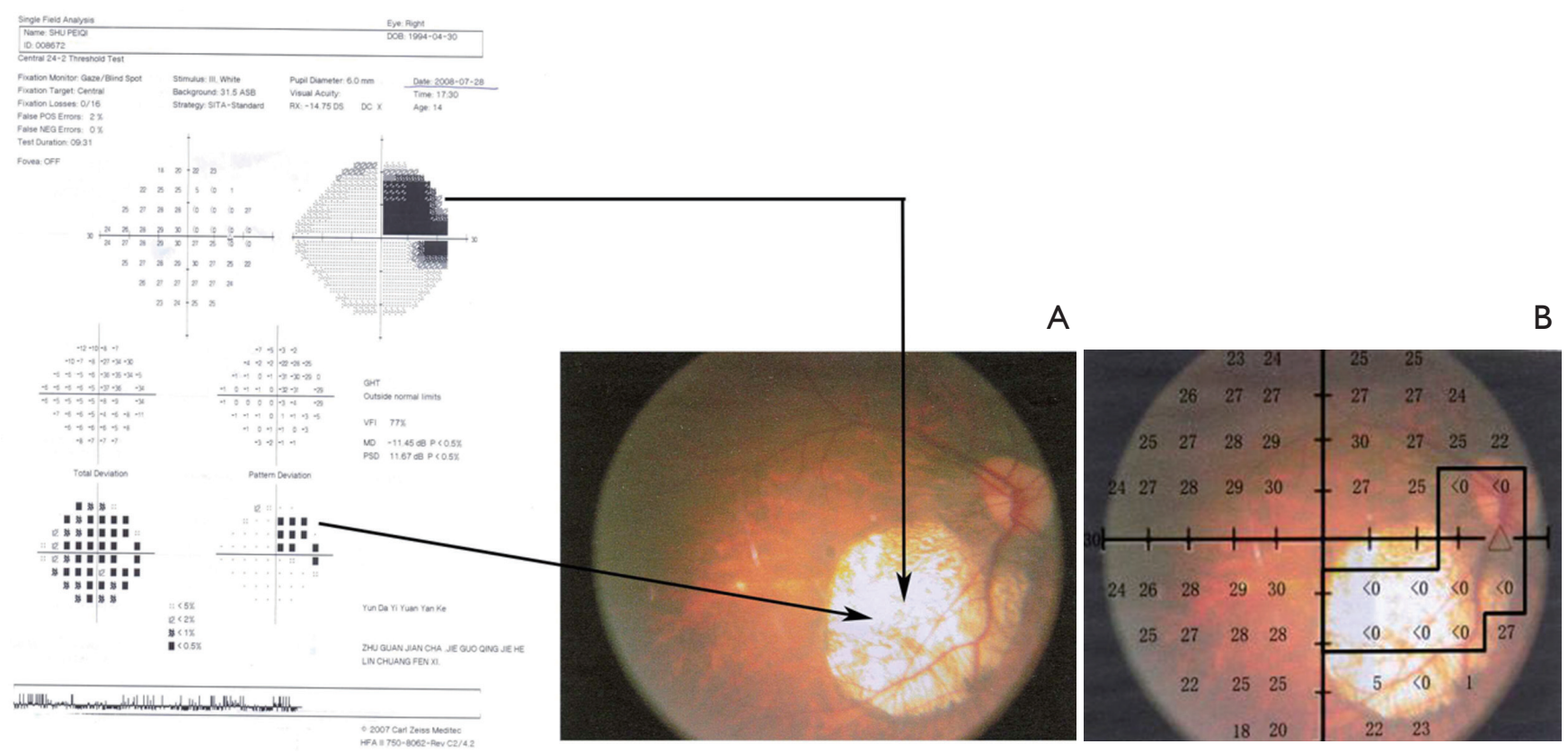

Figure 1 The morphology of scotomas in group II patients. (A) A sector-shaped defect in the superior temporal quadrant of the visual field and its corresponding macular atrophy, with a clear border in the inferior nasal quadrant of the ocular fundus; (B) macular atrophy in the inferior nasal quadrant of the ocular fundus and its corresponding retinal light sensitivity in the visual field defect.

accounting for $29.55 \%$, which included wedge-shaped visual defects, wing-shaped visual defects, superior arch-shaped scotomas coalescing into paracentral scotomas (Figure 1), sector-shaped defects coalescing into wedge-shaped defects, temporal sector-shaped defects (Figure 2), and superior temporal defects joining the nasal wedge-shaped defects (Figure 3).

A total of 203 scotomas were found in the visual fields of 13 eyes, 132 of which were relative scotomas $(65.02 \%)$.

\section{The frequency of scotomas in the four quadrants}

For each eye, 14 spots were examined in the superior and inferior nasal quadrant, respectively, and 13 spots were checked in the superior and inferior temporal quadrant, respectively. A total of 616 spots were checked in the superior and inferior nasal quadrant, while 572 spots were checked in the superior and inferior temporal quadrant in 44 eyes.

Regarding the frequencies of scotomas, 19.32\% (119 spots) were detected in the superior nasal quadrant, $16.07 \%$ (99 spots) in the inferior nasal quadrant, $25.87 \%$ (148 spots) in the superior temporal quadrant, and $38.74 \%$ (173 spots) were detected in the inferior temporal quadrant.

\section{Discussion}

The characteristics of the visual field in SM include a general 

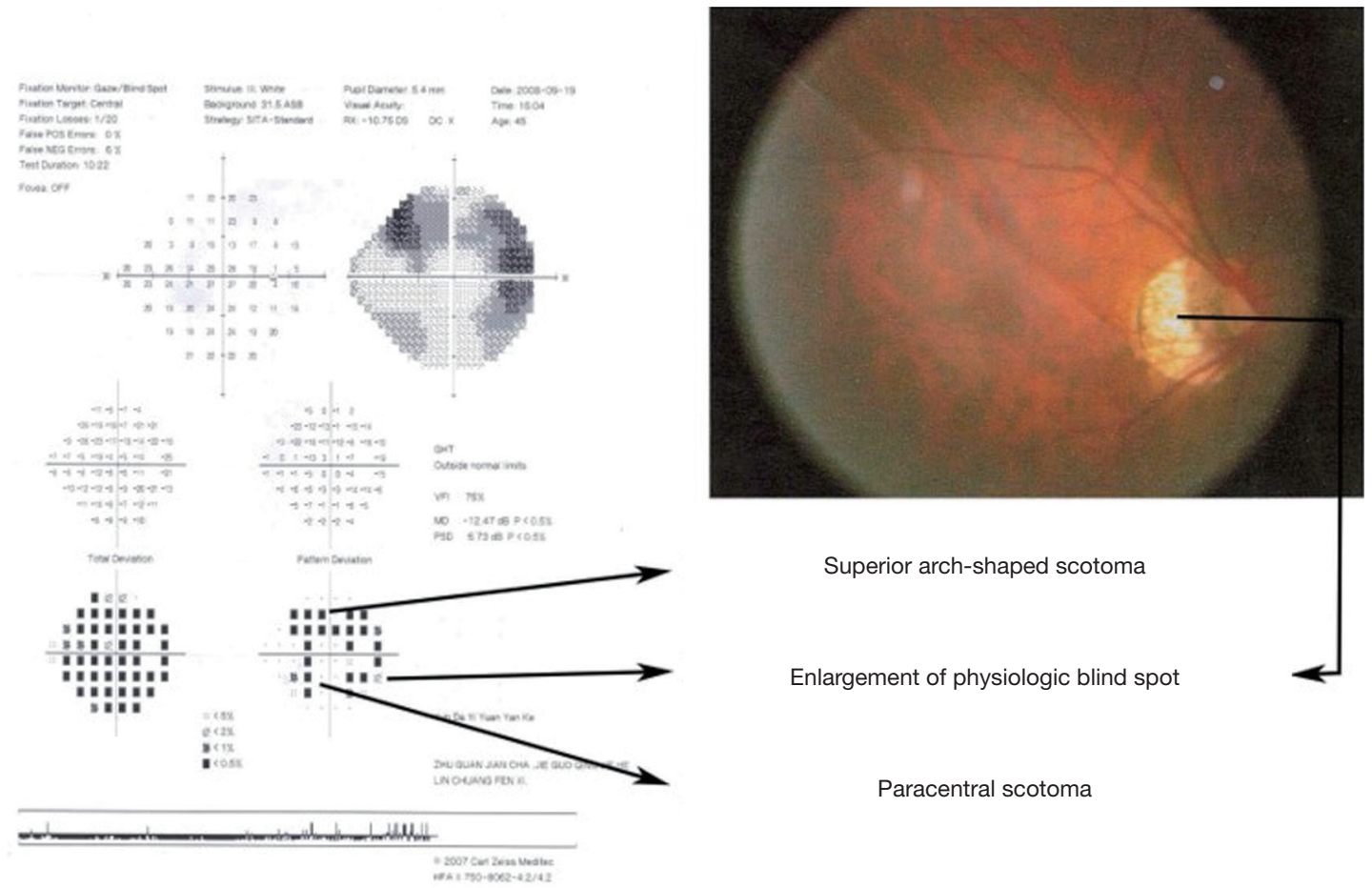

Paracentral scotoma

Figure 2 The morphology of scotomas in group III patients. Defects in the superior temporal quadrant coalescing into wedge-shaped visual defects in the nasal quadrant of the visual field and their corresponding geographic atrophy in the nasal quadrant; Fuchs spots are indicated in the temporal quadrant of the ocular fundus.

decrease in sensitivity, enlargement of the physiological blind spot, reduction of the peripheral visual field, and related central scotoma and/or paracentral scotoma. The myopic crescent near the optic disc was, in fact, parapapillary chorioretinal atrophy (PPCA), and patients suffering from HM with PPCA were likely to develop glaucoma $(6,7)$. Visual field defects caused by myopic crescents may be similar to glaucomatous visual field defects, or may gradually develop into focal retinal detachment near the optic disc (8). It was shown (9) that blood circulation became slow in the choroid of patients with HM, which led to hypoperfusion in the blood capillary of the choroid and the capillary system of the retina. As a result, the blood supply to the macular area was affected, and angiogenic factors were generated. This rendered the formation of neovascularization possible in the choroid, resulting in central vision loss in patients with HM, which manifested as central scotomas and paracentral scotomas (10).

The present study found that annular scotomas in the 44 eyes essentially occurred in the temporal quadrant. One possible reason for this may have been that the distance between the lamina cribrosa and retina in myopic eyes was observably shorter than in emmetropic or hyperopic eyes $(11,12)$. Therefore, complete optic disc cupping in patients with glaucoma and myopia is present only to half of the degree than in those with glaucoma, and not in those with myopia. The thin, tight lamina cribrosa diminishes the optic disc to prevent the damage caused by glaucoma. Consequently, the optic disc is particularly susceptible to damage by glaucoma. For a patient with SM and POAG, the pathological changes in the retina and choroid coexist with high IOP, which means that the optical nerve is attacked by "double forces". This will cause more damage to the optic ganglions, and at a more rapid pace.

The highest frequency of scotomas in the present study was observed in the inferior temporal quadrant, and these visual field defects were, to some degree, related to the normal distribution of optic nerve fibers. The average diameters of nerve fibers in the surrounding area of the superior quadrant and nasal quadrant were larger, which may eventually serve as an anatomical basis for explaining easy damage to the temporal visual field. This phenomenon was consistent with results reported by Vernon et al. (13), in which optical coherence tomography was used to reveal 


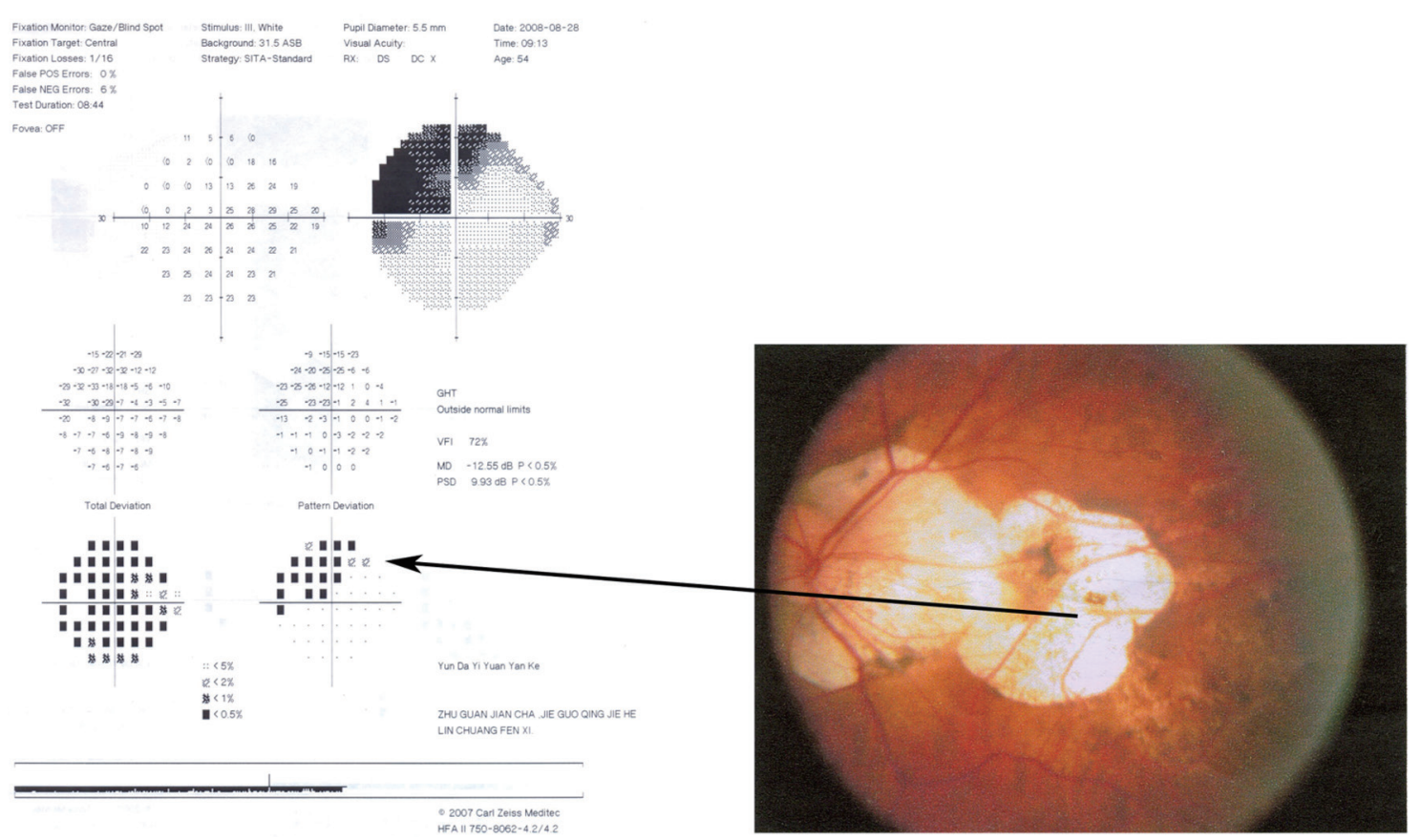

Figure 3 The morphology of scotomas in group IV patients. A superior arch-shaped scotoma joining the paracentral scotoma in the inferior, without any changes in the ocular fundus relating to the arch-shaped scotoma, but arc atrophy coalescing into the optic disc.

Table 2 Physiological changes in ocular fundus and forms of scotomas in visual field

\begin{tabular}{|c|c|c|c|c|c|}
\hline \multirow{2}{*}{ Forms of scotomas } & \multicolumn{5}{|c|}{ Groups of ocular fundus changes } \\
\hline & 1 & ॥ & III & IV & Total \\
\hline $\mathrm{N}$ & 8 & 14 & 7 & 15 & 44 \\
\hline Enlargement of physiological blind spot & 0 & 5 & 4 & 10 & $19(43.18 \%)$ \\
\hline Central scotoma & 0 & 5 & 3 & 9 & $17(38.64 \%)$ \\
\hline Nasal step scotoma & 0 & 2 & 3 & 3 & $8(18.18 \%)$ \\
\hline Arch-shaped scotoma & 0 & 1 & 0 & 0 & $1(2.27 \%)$ \\
\hline Annular scotoma & 0 & 2 & 0 & 1 & $3(6.82 \%)$ \\
\hline Temporal sector-shaped defect & 1 & 0 & 1 & 2 & $4(9.09 \%)$ \\
\hline
\end{tabular}

Notice: if there are multiple damages in the visual field, they are calculated respectively. For example, enlargement of physiological blind spot and temporal sector-shaped defect are found in the same visual field in Figure 3.

that the superior nervous fiber close to the optic disc among Caucasian HM patients was the thickest $(98.3 \mu \mathrm{m})$, while that in the nasal quadrant was the thinnest $(54 \mu \mathrm{m})$.

Relevant ocular fundus changes were found in $95.45 \%$ of the focal visual field defects in the present study. Among the 44 eyes, 13 eyes $(29.55 \%)$ developed multiple focal visual field defects. With the degeneration of ocular fundus changes in SM, extensive map-like atrophy may occur in the retina and choroid among patients in group IV. This correlated to an extensive decrease in sensitivity in the visual field and sector-shaped defects in the temporal quadrant. These defects conceal mild, scattered scotomas, which subsequently create larger defects by coalescing into one another. 
The above is different from arch-shaped or annular scotomas caused by the damage of arch-shaped nerve bundles in the Bjerrum area (damage of arch-shaped nerve bundles in the Bjerrum area results from glaucoma). The archshaped or annular scotomas caused by glaucoma appeared to be characterized by damage to the optic nerve, nasal step defects, and defects that correspond to the direction of the enlargement of the optic disk cup. Accordingly, it is suggested that relevant types of fundus damage should be detected for abnormal visual field defects (14). It is also conducive to identify factors (that may lead to visual field defects) that are related to causes other than glaucoma in their early stage; this can be performed by repeatedly examining the ocular fundus and visual field.

\section{Acknowledgments}

Funding: None.

\section{Footnote}

Reporting Checklist: The authors have completed the STROBE Reporting Checklist. Available at http://dx.doi. org/10.21037/apm-19-605

Data Sharing Statement: Available at http://dx.doi. org/10.21037/apm-19-605

Conflicts of Interest: All authors have completed the ICMJE uniform disclosure form (available at http://dx.doi. org/10.21037/apm-19-605). The authors have no conflicts of interest to declare.

Ethical Statement: The authors are accountable for all aspects of the work in ensuring that questions related to the accuracy or integrity of any part of the work are appropriately investigated and resolved. This study was conducted in accordance with the Declaration of Helsinki (as revised in 2013) and approved by the Ethics Committee of The First Affiliated Hospital of Kunming Medical University. A written informed consent was obtained from all participants (or their legal guardians).

Open Access Statement: This is an Open Access article distributed in accordance with the Creative Commons Attribution-NonCommercial-NoDerivs 4.0 International License (CC BY-NC-ND 4.0), which permits the noncommercial replication and distribution of the article with the strict proviso that no changes or edits are made and the original work is properly cited (including links to both the formal publication through the relevant DOI and the license). See: https://creativecommons.org/licenses/by-nc-nd/4.0/.

\section{References}

1. Iglesias AI, Ong JS, Khawaja AP, et al. Determining Possible Shared Genetic Architecture Between Myopia and Primary Open-Angle Glaucoma. Invest Ophthalmol Vis Sci 2019;60:3142-9.

2. Chen $\mathrm{M}, \mathrm{Wu} \mathrm{A}$, Zhang $\mathrm{L}$, et al. The increasing prevalence of myopia and high myopia among high school students in Fenghua city, eastern China: a 15-year population-based survey. BMC Ophthalmol 2018;18:159.

3. Wang WW, Wang HZ, Liu JR, et al. Diagnostic ability of ganglion cell complex thickness to detect glaucoma in high myopia eyes by Fourier domain optical coherence tomography. Int J Ophthalmol 2018;11:791-6.

4. Ikuno Y. Overview of the complications of high myopia. Retina 2017;37:2347-51.

5. Nitta K, Sugiyama K, Wajima R, et al. Is high myopia a risk factor for visual field progression or disk hemorrhage in primary open-angle glaucoma? Clin Ophthalmol 2017;11:599-604.

6. Jonas JB, Weber P, Nagaoka N, et al. Glaucoma in high myopia and parapapillary delta zone. PLoS One 2017;12:e0175120.

7. Miki A, Ikuno Y, Weinreb RN, et al. Measurements of the parapapillary atrophy zones in en face optical coherence tomography images. PLoS One 2017;12:e0175347.

8. Chen LW, Lan YW, Hsieh JW. The Optic Nerve Head in Primary Open-Angle Glaucoma Eyes With High Myopia: Characteristics and Association With Visual Field Defects. J Glaucoma 2016;25:e569-75.

9. Mo J, Duan A, Chan S, et al. Vascular flow density in pathological myopia: an optical coherence tomography angiography study. BMJ Open 2017;7:e013571.

10. Krishnan AK, Queener HM, Stevenson SB, et al. Impact of simulated micro-scotomas on reading performance in central and peripheral retina. Exp Eye Res 2019;183:9-19.

11. Sawada $Y$, Araie M, Ishikawa M, et al. Multiple Temporal Lamina Cribrosa Defects in Myopic Eyes with Glaucoma and Their Association with Visual Field Defects. Ophthalmology 2017;124:1600-11.

12. Miki A, Ikuno Y, Asai T, Usui S, Nishida K. Defects of the Lamina Cribrosa in High Myopia and Glaucoma. PLoS One 2015;10:e137909. 
13. Vernon SA, Rotchford AP, Negi A, et al. Peripapillary retinal nerve fibre layer thickness in highly myopic Caucasians as measured by Stratus optical coherence tomography. Br J Ophthalmol 2008;92:1076-80.

Cite this article as: Cao Q, Li L, Zhong H, Wei T, Yuan YS, $\mathrm{Li} \mathrm{Y}$. Changes to central visual fields in cases of severe myopia in a Chinese population. Ann Palliat Med 2020;9(5):2616-2622. doi: 10.21037/apm-19-605
14. Ding X, Chang RT, Guo X, et al. Visual field defect classification in the Zhongshan Ophthalmic Center-Brien Holden Vision Institute High Myopia Registry Study. Br J Ophthalmol 2016;100:1697-702. 